\title{
Zur quantitativen immunologischen Bestimmung von Serumproteinen
}

\author{
Von R. Lamerz, A. Fateh-Moghadam und M. KNeder ${ }^{1},{ }^{2}$ ) \\ I. Mediziniscbo Klinik (Direktor: Prof. Dr. med. H. Scbuiegk) der Universität Mïncbent tnd Klinisch-Cluemisches Instilut \\ (Chefarzt: Priv.-Doz. Dr. med. M. Knedel) am Städtischen Krankenbaus Mäincben-Harlacbing
}

(Eingegangen am 23. Januar/13. April 1973)

\begin{abstract}
Es wird über ein verbessertes Auswerteverfahren der einfachen radialen Immunodiffusion nach der Methode von MaNcrNr (Imnmunochemistry 2, 235-254 (1965)) anhand der Auswertung von 1053 Partigenplatten der Pa. Behringwerke bei 10 Scrumproteinen berichtet. Dic Auswertung erfolgte mit Hilfe einer Standardgeraden durch Erstellung einer linearen Regressionsgleichung aus den Standard-Konzentrationen mit zugehörigen Präzipitatsfächen. Aufgrund des dirckten Vergleichs der cinzclnen Regressionsgeraden für die verschiedenen Scrumproteine und unter Berücksichtigung der Chargen muß für jede Partigenplatte eine cigene Regressionsglcichung gefordert werden. Bei Prüfung der Genauigkeit der Methode ließ sich eine Gesamtstrcuung «wischen 2,4\% ('Transferrin) und 7,1\% (IgG) feststellen. Verschiedene andere Auswertungsverfahren erwicsen sich dem von uns beschriebenen als unterlegen. Neben diesen Befunden werden Tripartigenplatten (IgG, IgA, IgM) der Fa. Behringwerke und Immunoplates der Fa. Hyland für 8 Scrumproteine verglichen. Dabei zeigte sich, daß die auf Immunoplates durchgeführte Auswertung nach der Methode von FAuBx (J. Immunol. $94,84-90$ (1965), lineare Bezichung zwischen Durchmesser des Präzipitats und dem Logarithmus der Konzentration) der MaNcrNı-Methode (lineare Bezichung zwischen Präzipitatsfläche und der Konzentration) an Genauigkeit durchaus ebenbürtig und cbenso für Kleinrechner programmicrbar ist. Die im Vergleich zur MANcini-Methode auf Partigenplatten gefundenen Unterschicde waren nicht methodisch bedingt, sondern auf dic nicht vergleichbaren unterschiedlichen Konzentrationsangaben der Standards beider Firmen zurückzuführen.
\end{abstract}

\section{The quantitative immunological determination of serum proteins}

An improved cvaluation procedure for the simple radial immunodiffusion method of MANCrNI (Immunochemistry 2, 235-254 (1965)) is reported for 10 serum proteins, using 1053 Partigen plates from Behringwerkc. A calibration line was cstablished from the linear regrcssion of the standard concentration and the corresponding arcas of precipitation. A regression analysis was performed for each Partigen plate by the direct comparison of the individual regression lines for the different serum proteins, taking the differences between batches into account. The results showed a scattcr of between $2.4 \%$ (transferrin) and $7.1 \%(\mathrm{IgG})$. Other methods of evaluation were found to be less accuratc. The Tripartigen plates (IgG, $\operatorname{lgA}, \mathrm{IgM}$ ) from Behringwerke and Immunoplates from Hyland were also compared for 8 serum proteins. It was found that the evaluation on Immunoplates by the method of FAFIEY (J.Immunol. 94, 84-90(1965)) (linear relationship between diameter of precipitation and logarithm of the concentration) and by the MANCINI method (linear relationship between precipitation area and concentration) are equally accurate, and both can be programmed on a calculating machinc. The method on Partigen plates showed differences from the MANCINI method, which were not due to a lack of agreement between the two methods, but to differences in the stated concentrations of the standards from the two firms.

Die Methode der einfachen radialen Immunodiffusion wird in zwei Modifikationen angewandt, für die auch handelsübliche Immunodiffusionsplatten erhältlich sind. Nach den Verfahren von MaNcinI (1) besteht zwischen der terminalen Präzipitationsfläche und der Antigenkonzentration eine lineare Beziehung. Nach diesem Verfahren werden die Partigenplatten der Fa. Behringwerke ausgewertet. Die Modifikation von FAHEY (2) nützt die lineare Beziehung zwischen dem PräzipitatsDurchmesser eines noch diffundierenden Antigens und dem Logarithmus der Antigenkonzentration aus. Eine Anwendung dieses Verfahrens erfolgt mit den handelsüblichen Immunoplates der Fa. Hyland/Travenol.

In der vorliegenden Arbeit wird übcr unsere Erfahrungen mit der einfachen radialen Immunodiffusionsmethode nach MANCINI in der Mikromodifikation von Augener (3) anhand der Auswertung von 1050 handelsüblichen Partigen-Platten berichtet, die im Vergleich mit selbst hergestellten Platten eine gute Úbereinstimmung zeigten (4). Dabei wird ein verbessertes und vereinfachtes Auswerteverfahren unter Verwendung eines Mcß-Mikroskops und einer elektronischen
Rechenmaschine beschrieben. Ferner wird ein Vergleich mit Tripartigen-Platten sowie Immuno-Plates der Fa. Hyland/Travenol und derer Standards gezogen.

\section{Methodik}

\section{Prinzip}

Im Prinzip diffundieren bei der cinfachen radialen Immunodiffusion dic Antigenmoleküle radial von einem runden Startloch aus in eine mit homologem spezifischen Antiserum vermischte Agargelschicht, wobei sie einen mit der Zeit konzentrisch wachsenden Präzipitationszylinder bilden, dessen innercr Rand vom Antigenbchälter gebildet wird und dessen äußcrc Bcgrenzung der besonders gut sichtbarc Präzipitationsring darstellt. Dieser wandert konzentrisch bis zur Ausbildung eincs stabilen Glcichgewichts der Aquivalenz von Antigen- und $\Lambda$ ntikörperkonzentration d. h. bis alles diffundicrende Antigen vom homologen Antikörper gebunden ist.

Nach der Modifikation von MANCINI steht dic nach vollständig beendeter Diffusion erreichte terminale Präzipitatsfläche in direktcr linearer Bezichung zur vorgegebenen Antigenmenge:

1) Die Autoren sind Mitglieder des SFB 37 „Restitution und Substition innerer Organc" München.

2) Das hier verwendete Konzentrationsmaß $10^{-2} \mathrm{~g} / \mathrm{I}$ entspricht ing/100 ml. 
$\mathrm{F}_{\mathrm{G}}=\mathrm{F}_{\mathrm{O}}+\mathrm{cQ}_{\mathrm{Ag}}$

$\mathrm{F}_{\mathrm{G}}=$ Präzipitatfläche + Antigen-Reservoir-Fläche

$\mathrm{Q}_{\mathrm{Ag}}=$ Antigenmenge.

Die grafische Darstellung der.Funktionsgleichung ist ëine Gerade mit der Steigung $\mathrm{c}$ und dem Ordinatenabschnitt $\mathrm{F}_{\mathrm{o}}$.

Nach den Untersuchungen von MANcINI (1) ist die Steigung c eine Funktion der Antiserumkonzentration. Je höher die verwendete Antikörperkonzentration, desto kleiner die Steigung c. Der Wert $F_{0}$ (Ordinatenabschnitt) hängt mit der Größe der Antigenbehälterfläche zusammen: Je größer das Antigen-Reservoir, um so größer auch der $\mathrm{F}_{0}$-Wert. Auch wachsende Lösungsmittelvolumina führen $\mathrm{zu}$ einem Anstieg des $\mathrm{F}_{0}$-Wertes. Die Zeit bis zum Erreichen der terminalen Präzipitatsfläche ist u. a. vom Molekulargewicht des untersuchten Antigens und damit von seinem Diffusionskoeffizienten abhängig: die bis dahin verstrichene Diffusionszeit hängt auch von der Temperatur ab: eine höhere Temperatur bewirkt ein schnelleres Wandern der Antigenmoleküle.

Nach Mancin ließ sich die Empfindlichkeit der einfachen radialen Immunodiffusion zu $10-20 \mu \mathrm{g} / 1$ bestimmen. Die Gesetzmäßigkeiten der MANCINI-Modifikation treffen zum Teil auch für die FAHEY-Modifikation zu. Hier besteht eine lineare Beziehung $z$ wischen dem zu einem bestimmten Zeitpunkt während der Diffusion gemessenen Präzipitatdurchmesser und dem Logarithmus der Antigenkonzentration. $\mathrm{Da}$ jedoch unter Diffusion gemessen wird, können entsprechende Platten nach oft wesentlich kürzeren Diffusionszeiten abgelesen werden.

Vorbereitung und Beschickung der Platten

Zur Füllung der Antigenbehälter verwendeten wir eine HamiltonMikroliterspritze mit Dosiervorrichtung. Eingefüllt wurden genau $2 \mu \mathrm{l}$ Standard- bzw. Analysenlösung in die Partigen- sowie $5 \mu \mathrm{l}$ in die Tripartigen- bzw. M-Partigen-Platten und die vergleichsweise untersuchten Immuno-Plates der Fa. Hyland. Zur Herstellung einer Konversionsgeraden wurden 3-4 unterschiedliche Standardserum-Verdünnungen pro Platte benutzt. Nach dem Füllen der Antigen-Reservoirs mit Standard- bzw. Analysenlösung wurden die Platten in einer feuchten Kammer bei Raumtemperatur auf einer horizontalen Ablage für 24 bzw. 48 h (z. B. höhermolekulare Antigene $\operatorname{IgM}, \alpha_{2}$-Makroglobulin) aufbewahrt und im

$$
\begin{aligned}
& \text { Methode nach Mancini } \\
& r=\frac{\sum x y-N \bar{x} \bar{y}}{\sqrt{\left(\Sigma x^{2}-N \bar{x}^{2}\right)\left(\Sigma y^{2}-N \bar{y}^{2}\right)}} \\
& -1 \leqq r \leqq 1 \\
& A=\bar{y}-B \bar{x} \\
& B=\frac{\sum x y-N \bar{x} \bar{y}}{\Sigma x^{2}-N \bar{x}^{2}} \\
& x=-\frac{A}{B}+\frac{1}{B} y \\
& \text { Konzentration Fläche } \\
& x \text { : Konzentration in } \mathrm{mg} / 100 \mathrm{~m} \\
& d=b-a \quad 0=c \\
& F p=\left[\frac{\pi}{4} c^{2}-(b-a)^{2}\right] \\
& F \bar{p}=\frac{F p_{1}+F p_{2}}{2}=\frac{\sum_{i=1}^{N} F p_{i}}{N}
\end{aligned}
$$
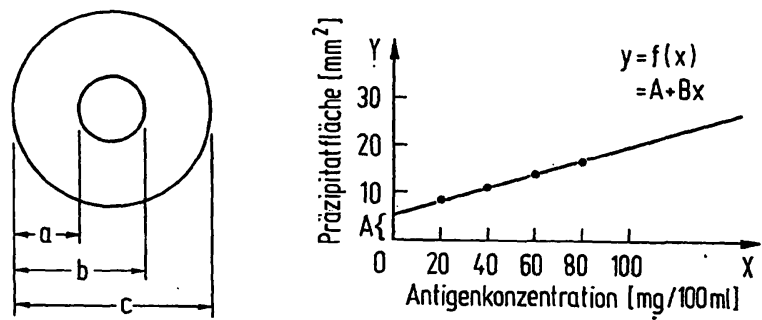

Anschluß daran zur Präzipitatsmessung herangezogen. Bei den im Vergleich untersuchten Immuno-Plates betrugen die Diffusionszeiten $4 \mathrm{~h}$ bei $37^{\circ} \mathrm{C}$ für IgG, Transferrin und Albumin sowie $16 \mathrm{~h}$ bei Raumtemperatur für Coeruloplasmin, $\alpha_{2}$-Makroglobulin, $\beta_{1 \mathrm{~A}}$-Globulin, IgA und IgM.

\section{Konzentrationsbereiche}

Die für die Serumproteinbestimmung auf ImmunodiffusionsPlatten brauchbaren Konzentrationsbereiche gehen aus Tabelle 1 hervor.

\section{Auswertung}

Für die Auswertung der Partigen- bzw. Tri- oder M-PartigenPlatten muß nach beendeter Diffusion die terminale Präzipitatsfläche oder das Quadrat des Durchmessers bestimmt werden. Wir bestimmen die exakte Präzipitationsfläche aus den gemessenen drei Teilstrecken des Durchmessers a, b, $c$ und den darauf senkrecht stehenden Teilstrecken $a^{\prime}, b^{\prime}$ und $c^{\prime}$ (Abb. 1). Diese Werte wurden in ein Auswerteformular zusammen mit den Konzentrationen der Standards und den Bezeichnungen der Analysen,

Tab. 1

Konzentrationsbereiche bei der linearen radialen Immunodiffusion in $10^{-2} \mathrm{~g} /$

\begin{tabular}{lcc}
\hline \multicolumn{1}{c}{ Protein } & Partigen-Platten & Immunoplates \\
\hline Albumin & $15-40$ & $18-710$ \\
$\alpha_{1}$-Antitrypsin & $10-40$ & \\
$\alpha_{2}$-Haptoglobin & $10-60$ & \\
$\alpha_{2}$-Makroglobulin & $15-80$ & $65-390$ \\
Coeruloplasmin & $5-20$ & $9,5-55$ \\
Transferrin & $15-40$ & $80-440$ \\
$\beta_{1 A}$ - $r$ lobulin & $5-40$ & $60-360$ \\
IgG & $10-40$ & $280-2000$ \\
& $10-120^{*}$ & \\
IgA & $10-40$ & $60-400$ \\
IgM & $10-100^{*}$ & \\
& $5-30$ & $18-130$ \\
\hline
\end{tabular}

* Tripartigen

\section{Methode nach Fahey}

Korrelationskoeffizient Standardgerade (lineare Regressionsgerade)

$$
r=\frac{\sum x y-N \bar{x} \bar{y}}{\sqrt{\left(\Sigma x^{2}-N \bar{x}^{2}\right)\left(\Sigma y^{2}-N \bar{y}^{2}\right)}} \quad \begin{array}{ll}
A=\bar{y}-B \bar{x} \\
-1 \leqq r \leqq 1 & B=\frac{\sum x y-N \bar{x} \bar{y}}{\Sigma x^{2}-N \bar{x}^{2}} \\
x=-\frac{A}{B}+\frac{1}{B} y
\end{array}
$$

log.Konzentration Durchmesse
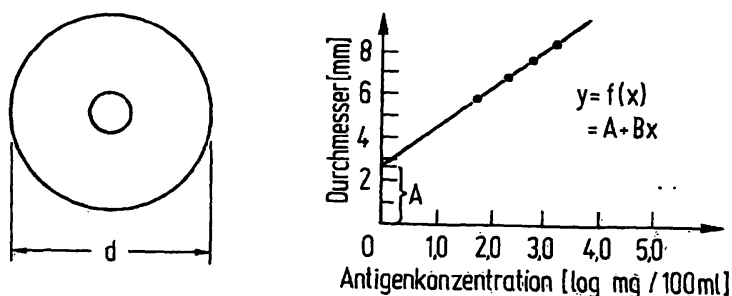

1.Logarithmieren der Standard-Konzentrations-Werte

2.Berechnung der linearen Regressionsgeraden

3.Entlogarithmieren der logarithmierten Konzentrationswerte

Abb. 1

Quantitative immunologische Proteinbestimmung nach der einfachen radialen Immunodiffusion. Vorgehen bei der Berechnung nach der Auswertemethode von MANCINI bzW. FAHEY 

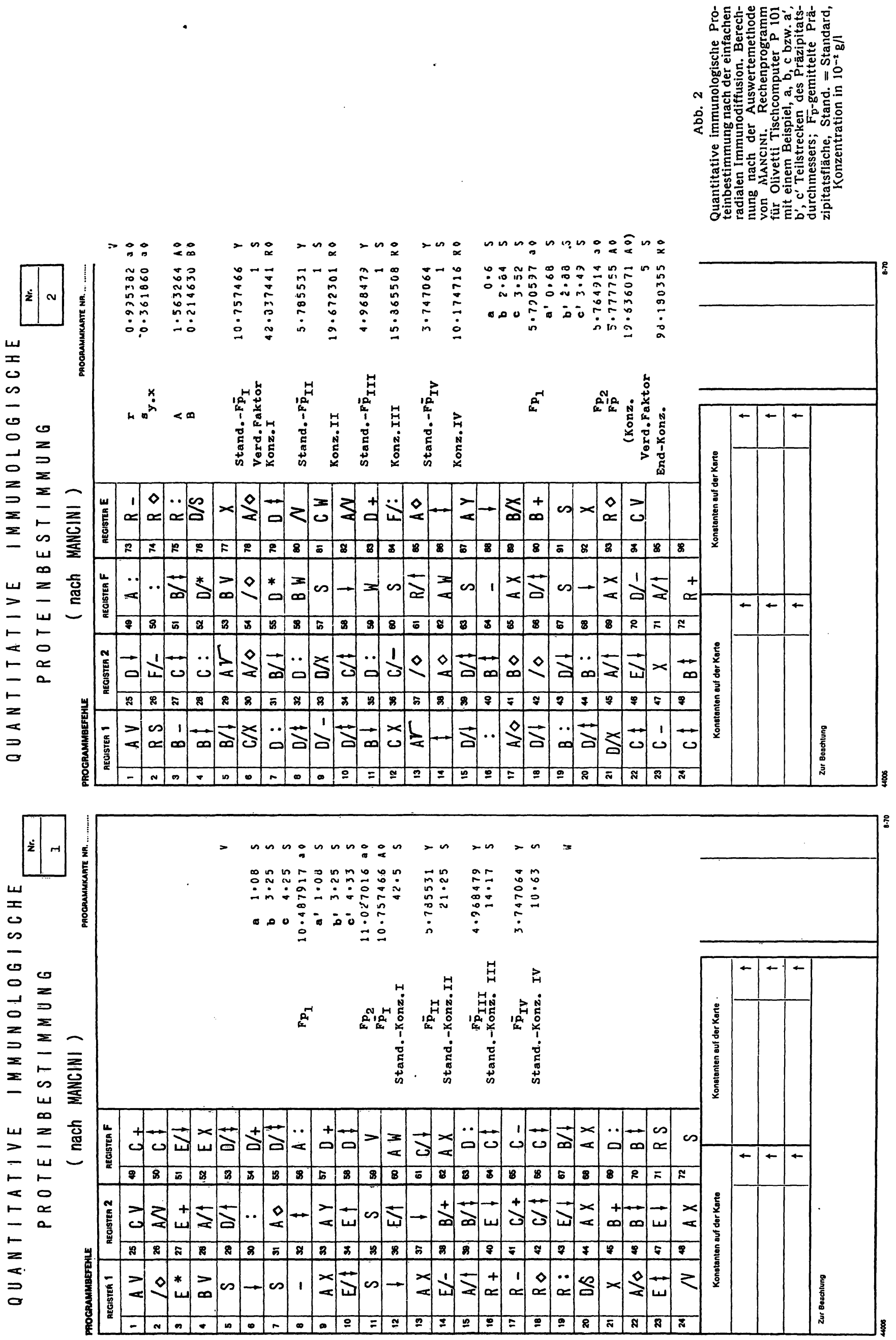
Chargen-Nr. und Patientenserum-Verdünnungsfaktoren eingetragen.

Für dic Auswertung der Immuno-Plates nach dem FaHEYschen $\checkmark c r f a h r e n$ werden lediglich zwei. einfache senkrecht aufeinanderstchende Durchmesser der Präzipitatstinge zu den festgesetzten Zeitpunkten gemessen.

Die Messungen wurden mit Hilfe eines Leitz-Meßkomparators und eines von uns entwickelten Dunkelfeldadapters durchgeführt.

Ublicherweise trägt man die zusammengehörigen Wertepaare: Präzipitatsflächenwert oder Quadrat des Durchmessers mit zugchöriger Standard-Konzentration (Partigen-Platten) bzw. Durchmesser und zugehörigen Logarithmus der Standard-Antigenkonzentration (Immuno-Plates) auf linearem mm-Papier auf. Nach der FAfEY-Methode kann man auch die Antigenkonzentration auf dem logarithmischen Schenkel und den Durchmesser auf dem linearen Schenkel eines semilogarithmischen mm-Papiers auftragen. Anschließend legt man durch die erhaltenen Punkte eine „von Auge" angepaßte Gerade. An dieser Bezugsgeraden werden dann die Konzentrationen der untersuchten Analysenlösungen innerhalb der niedrigsten und höchsten StandardPunkte abgelesen.

\section{Reclonerisclbes Verfabren}

Die mehr oder weniger subjektive Anpassung an die Standardpunkte läßt sich aber durch Ermittlung einer linearen Regressionsgeraden für diese Punkte nach dem Prinzip der kleinsten Abweichungsquadrate besser durchführen.

Sämtliche statistischen Auswertungen ( $t-T$ est für Paardifferenzen, $\%^{2}$-Vierfeldertest, Mittelwert, Standard-Abweichung, Variationskoeffizient, lineare Regressionsgerade, Korrelationskoeffizient (5)) sowie die Berechnung der Serumkonzentration wurden an einem Olivetti Tischcomputer Programma P 101 mit zumeist eigenen Programmen durchgeführt. Das von uns für die Partigen- bzw. Tripartigen-Platten entwickelte Auswerteprogramm (Abb. 2) führt folgende Rechenoperationen durch:

Pro Präzipitat werden 2 Flächenwerte nach der in Abbildung 1 wiedergegebenen Formel aus den gemessenen Werten a, b, $c$ bzw. $a^{\prime}, b^{\prime}$ und $c^{\prime}$ berechnet, gemittelt und zusammen mit den zugehörigen drei bis vier Standard-Konzentrationswerten zur Berechnung der Regressionsgeraden verwandt. Die Kennwerte der Regressionsgleichung

$y=A$ (Ordinatenabschnitt) + B (Steigung der Geraden) $\cdot x$ werden daraufhin errechnet und zur weiteren Bestimmung von Ordinatenbeispielen gespeichert. Als zusätzliche Kenngrößen werden noch der lineare Korrelationskoeffizient $\mathrm{r}$ und der $\mathrm{s}_{\mathrm{y}} \cdot \mathrm{x}$-Wert (Standardfehler der y-Schätzung) ausgedruckt.

In der Abbildung 2 ist auch ein Rechenbeispiel wiedergegeben. Daraus geht hervor, daß im Anschluß an den unbedingten Sprungbefehl "V" nach Eingabe der gemessenen Teilstrecken eines Standard-Präzipitates jeweils der berechnete Flächenwert und nach zivei Flächenwerten eines Standard-Präzipitates der zugehörige Mittelwert automatisch ausgedruckt wird. Nach Eingabe der zugehörigen Standardkonzentrationen wiederholt sich das Rechenschema für die Standards II bis III bzw. IV. Die Standardwerte können auch getrennt direkt mit dem entsprechenden gemittelten Präzipitatflächenwert eingegeben werden. Schließlich wird das Programm mit dem unbedingten Sprungbefehl „W" unterbrochen und die zweite Programmkartenhälfte ohne Löschung eingegeben. Ein neuer Sprungbefehl "V“ führt zu Berechnung und Ausdruck des Korrelationskoeffizienten $r$, des $s_{y} \cdot x$-Wertes sowie der Kennwerte für die lineare Regressionsgleichung $A$ und B. Nun werden nach der berechneten Regressionsgleichung unter Eingabe der zuvor gemittelten Präzipitatflächen der Standards die zugehörigen Konzentrationen als Ordinatenbeispiele der linearen Regressionsgleichung berechnet und mit den vorgegebenen Ausgangskonzentrationen der Standards verglichen. Erst dann wird entschieden, ob die berechnete Regressionsgleichung akzeptabel ist. Nun werden dic Präzipitatflächen und -konzentrationen der Patientenseren (Analysenlösungen) berechnet. Vor dem endgültigen Ausdruck der Konzentrationen können durch Eingabe des zugehörigen Verdünnungsfaktors diese noch entsprechend multipliziert werden. Insgesamt dauert die Auswertung einer einzigen Partigen-Platte 3-5 min und liefert am Schluß einen übersichtlich angeordneten Rechenstreifen, der zusammen mit dem Auswerteformular dokumentationsgerecht abgeheftet werden kann.

Im Prinzip wurde bei der Auswertung der Immuno-Plates nach der FAHEY-Methode ähnlich verfahren. Mit von uns entwickelten Rechenprogrammen werden zunächst die Standard-Konzentrationen logarithmiert, und dann zusammen mit zwei aufeinander senkrecht stehenden gemessenen Durchmessern der zugehörigen Präzipitate in das Auswerteprogramm eingegeben. Daraufhin erfolgt die Berechnung der linearen Regressionsgeraden y (Durchmesser) $=\mathrm{A}+\mathrm{B} \cdot \mathrm{x}$ (logarithmierte Antigen-Konzentration) und des zugehörigen Korrelationskoeffizienten. Die dann berechneten Logarithmen der Analysen-Antigen-Konzentrationen werden automatisch entlogarithmiert und nach Multiplikation mit dem Verdünnungsfaktor als Endkonzentration ausgedruckt.

\section{Ergebnisse und Diskussion}

\section{Präzision der Methode auf Partigen-Platten}

\section{Messung}

Beim Vergleich der Messungen zwischen Meß-Mikroskop, -Lupe und-Schablone im $t$-Test für Paardifferenzen konnten signifikante Meßwertunterschiede gefunden werden. Sie waren für die Quadrate der bestimmten Durchmesser deutlicher als bei Berücksichtigung der errechneten Konzentrationen. In einer weiteren Versuchsanordnung wurde die individuelle Meßstreuung bei der Auswertung einer Partigen-Platte von 5 Versuchspersonen untersucht. Die Variationskoeffizienten schwankten bei Bestimmung des Durchmesserquadrats zwischen 1,4 und $4,4 \%$, bei Berücksichtigung der anhand der jeweiligen Regressionsgeraden bestimmten Konzentrationen jedoch nur zwischen 0,3 und 2,1\%.

\section{Regressionsgerade}

Ein Maß für die Genauigkeit, mit der die zur Auswertung verwandten Standardpunkte um die Regressionsgerade streuen, ist die Größe des Korrelationskoeffizienten $\mathrm{r}$. Er sollte mindestens einen Wert von $>0,95$, besser aber von $>0,98$ betragen. Als weiterer Parameter zur Beurteilung der Regressionsgeraden beachten wir neben dem $\mathrm{s}_{\mathrm{y} \cdot \mathrm{x}}$-Wert den Grad der Konzentrationsabweichung vom vorgegebenen Standardwert, wenn man die nicht an die Regressionsgerade angepaßten gemessenen Flächenwerte der Standardpunkte zur Konzentrationsberechnung verwendet. Je größer der Wert von $r$, desto geringer ist die Abweichung vom erwarteten Konzentrationswert.

\section{Vergleich der Regressionsgeraden mebrerer Partigen-Platten}

Es wurde untersucht, ob die für eine Partigen-Platte berechnete Regressionsgerade auch für andere Platten des gleichen Proteins verwendbar ist. Dazu wurden die Eichgeraden für 10 Serumproteine von insgesamt 881 einfachen $20 \mathrm{M}$ - und 152 Tri-Partigen-Platten mit einem Korrelationskoeffizienten über 0,95 auf die Streuung ihrer Kennwerte A (Ordinatenabschnitt) und $B$ (Steigung) verglichen.

Es ließ sich zeigen, daß die Streuung um den Mittelwert von A extrem hoch liegt, während sie für die Steigung $B$ deutlich geringer, aber ausgeprägt bestand (Vari- 
Tab. 2

Vergleich von 5 Auswertungsverfahren der einfachen radialen Immunodiffusion nach Mancins. Mittelwert (z), Standardabweichung (s) und Varlationskoeffizient $(V \%)$ von Mehrfachbestimmungen bei Auswertung derselben Partigenplatte nach Verfahren a, b, $c$, d und $c$ (siehe Text). - significante Unterschiede der Varianzen ( $\left(^{2}\right)$ zur Varianz der Methode c. Konzentration in $10^{-2} \mathrm{k} / \mathrm{l}$

\begin{tabular}{|c|c|c|c|c|c|c|c|}
\hline & \multicolumn{2}{|l|}{ Protein } & \multirow{3}{*}{$\begin{array}{c}a \\
1449,3 \\
101,2\end{array}$} & \multirow{3}{*}{ 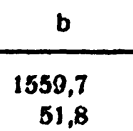 } & \multirow{2}{*}{$\frac{c}{1490,5}$} & \multirow{2}{*}{$\frac{d}{1502,3}$} & \multirow{2}{*}{$\frac{e}{1514,1}$} \\
\hline & IgO & $\mathbf{z}$ & & & & & \\
\hline & $n=6$ & 8 & & & 86,0 & 59,1 & 39,8 \\
\hline & & $v \%$ & $7,0 \%$ & $3,3 \%$ & $5,8 \%$ & $3,9 \%$ & $2,5 \%$ \\
\hline & IgA & 8 & 232,6 & 255,7 & 254,1 & 252,0 & 245,9 \\
\hline & $n=6$ & 8 & 22,0 * & $11,1 *$ & $9,4 *$ & 4,7 & 3,9 \\
\hline & & $V \%$ & $9,5 \%$ & $4,4 \%$ & $3,7 \%$ & $1,9 \%$ & $1,6 \%{ }^{\circ}$ \\
\hline & $\lg M$ & $x$ & 119,5 & 120,8 & 125,7 & 125,1 & 117,2 \\
\hline & $n=5$ & 8 & 5,1 & 4,8 & 5,2 & 4,5 & 4,1 \\
\hline & & $v \%$ & $4,3 \%$ & $4,0 \%$ & $4,1 \%$ & $3,5 \%$ & $3,4 \%$, \\
\hline & Albumin & X & 2309,3 & 2421,3 & 2389,3 & 2373,9 & 2375,8 \\
\hline & $n \ldots 4$ & 8 & 72,9 & 37,5 & 34,1 & 33,9 & 30,0 \\
\hline & & $V \%$ & $3,2 \%$ & $1,6 \%$ & $1,4 \%$ & $1,4 \%$ & $1,2 \ldots$ \\
\hline & Cocruloplasmin & $\mathrm{x}$ & 43,5 & 40,6 & 40,5 & 40,1 & 30,2 \\
\hline & $n \div 6$ & B & $5,5^{*}$ & 1,5 & 1,5 & 1,1 & 1,1 \\
\hline \multirow[t]{7}{*}{ - } & & $V \%$ & $12,6 \%$ & $3,6 \%$ & $3,6 \%$ & $2,8 \%$ & $2,8 \ldots$ \\
\hline & Transferrin & 8 & $206, \theta$ & 221,7 & 229,5 & 228,2 & 225,$6 ;$ \\
\hline & $n=6$ & 8 & 12,6 & 12,9 & 16,5 & 16,6 & 17,5 \\
\hline & & $v \%$ & $6,1 \%$ & $5,8 \%$ & $7,2 \%$ & $7,3 \ldots$ & $7,8 \%$ \\
\hline & $\beta_{1} \wedge-$ Olobulin & $\mathbf{x}$ & 128,9 & 125,1 & 125,3 & 117,0 & 114,3 \\
\hline & $n=8$ & 8 & $11,8^{*}$ & $8,7^{*}$ & $5,4^{*}$ & $3,6^{*}$ & 1,7 \\
\hline & & v\% & $9,2 \%$ & $6,9 \%$ & 4,3\% & $3,1 \%$ & $1,4 \%$ \\
\hline
\end{tabular}

ationskoeffizient $\mathrm{V}$ zwischen 9,6 und $75 \%$ ). Berücksichtigt man nur die Platten gleicher Charge, (9 Scrumproteine, insgesamt 16 Chargen) ändert sich das Ausmaß der Streuung der $\mathrm{A}-\mathrm{Werte}$ kaum, während dic Streuung der Steigungen $B$ deutlich abnimmt (V \%wischen 6,3 und $24,8 \%$ ). Aus diesen Untersuchungen folgt, daß für jede einzelne Partigen-Platte eine eigene Regressionsgerade crmittelt werden muß.

\section{$V$ ergleich verscbiedener Auswerteverfabren}

Pünf unterschiedliche Auswerteverfahren wurden mitcinander verglichen (Tab. 2):

a) Messung des Durchmessers mit Mcß-Schablone und grafische Auswcrtung

b) Messung des Durchmessers mit Meß-Mikroskop und grafische Auswertung

c) Messung des Durchmessers mit Meß-Mikroskop und Kon\%entrationsberechnung mit Computer ( $y$ : Durchmesser)

d) Messung des Durchmessers mit Mcß-Mikroskop und Konzentrationsberechnung mit Computer ( $y$ : Quadrat des Durchmessers)

c) Messung des Durchmessers mit Meß-Mikroskop und Konzentrationsberechnung mit Computcr nach dem eigenen Verfahren ( $y$ : Präripitatsfläche).

Nach Tabclle 2 nimmt bei Vergleich von Mchrfachbestimmungen die Streuung b\%w. der Variationskocffizient von a nach $c$ in der Regel ab. Die Zahlen mit Sternchen weisen auf einen signifikanten Unterschicd zur Methode e hin.

\section{Eigenes Auswerteverfabren}

Zur Bestimmung der Präzision der Methode bei Anwendung des eigenen Auswertcverfahrens wurden dic Konzentrationen aus Mehrfachbestimmungen von Stan-
dardscrum-Verdünnungen für 10 Scrumprotcinc in 3-4 Kon\%entrationen von 470 Partigen-Platten (312 einfachen, 138 Tri-, 20 M-Partigen) mit cincm Korrclationskocffizienten von 0,98 und gröfjer mitcinander verglichen (Tab. 3). Dabei \%eigte sich, daß die Streuung der Mittclwerte mit \%unchmender Verdünnung ansteigt. Dic Gesamt-Variationskoeffirienter aller Standardscrum-Verdünnungen nach Rückrechnung auf dic Ausgangskonzentration schwankten für die untersuchten Proteine zwischen 2,4\%, (Transferrin) und $7,1 \%$ (IgG). Die berechneten Mittelwerte selbst weichen kaum von den erwarteten Werten ab.

\section{Auswertung auf Tripartigenplatten}

Die seit einiger Zcit im Handel befindlichen TripartigenPlatten verfügen über cinen größeren Antikörpergehalt und erlauben dic Einfüllung größerer Volumina $(5 \mu \mathrm{l})$. Nach Angabe der Hersteller sollen sie für normale JgA- und IgM-Spiegcl mit unverdünntem sowic für normale IgG-Spicgel mit 1:10 verdünntem Serum beschickt werden können.

Ein wichtiger Vortcil für Routine-Untersuchungen ist, daß laut Angabe der Hersteller auf dic Erstellung cincr Ejchgeraden verzichtet werden kann, da wegen des standardisierten Antikörpergchalts sowie präziscr Rcproduzierbarkeit von Antigen-Reservoir und Schichtdicke anhand einer mitgegebenen Konversionstabelle dirckt von jedem beliebigen Durchmesserquadrat des Präzipitats auf dic zugehörige Konzentration geschlossen werden kann. Dic Überprüfung der Brauchbarkeit der einzelnen Platten jst heute durch Verwendung von Präzisions- und Richtigkeitskontrollscren möglich. Für besonders exakte Bestimmungen sind aber auch für dic 'Tripartigen-Platten Standard-Immunglobuline für je drei verschiedene Konzentrationen crhältlich. 
Tab. 3

Präzision der einfachen radialen Immunodiffusion nach MANciNı auf Partigenplatten (Fa. Behringwerke) aus Mehrfachbestimmungen von Standardprotein-Lösungen in 3-4 Konzentrationen. Unter unv. wurden die Konzentrationen sämtlicher Standardverdünnungen auf die unverdünnte Alısgangskonzentration zurückgerechnet und gemittelt. (Ausnahme: *Tripartigen-IgG-IgA, -IgM und Haptoglobin-M-Partigen). Mittelwert $\overline{\mathbf{x}}$, Standardabweichung s, Variations-Koeffizient V. Konzentrationen in $10^{-2} \mathrm{~g} / \mathrm{l}$

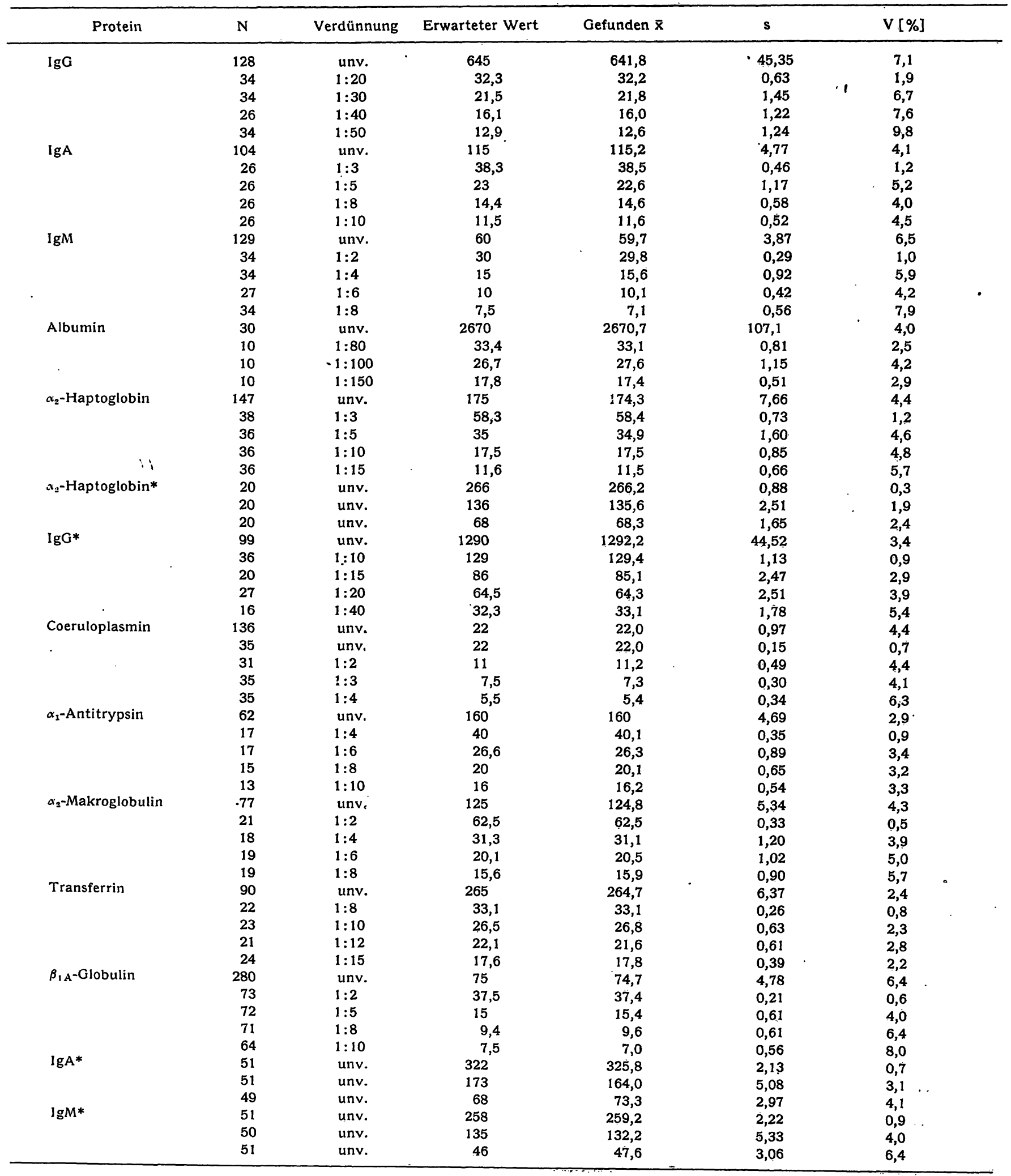

Wir untersuchten 4 IgG-, IgA- und IgM-TripartigenPlatten mit einer Standard-Protein-Verdünnnungsteihe und verglichen die berechneten Regressionsgeraden mit den Geradengleichungen der vorgegebenen Eichkurven bzw. -tabellen. Ein Beispiel der grafischen Darstellung der Auswertung einer IgG-Tripartigen-Platte zeigt Abbildung 3. Für IgG und IgM ließ sich eine gute Übereinstimmung unserer Regressionsgleichungen mit 


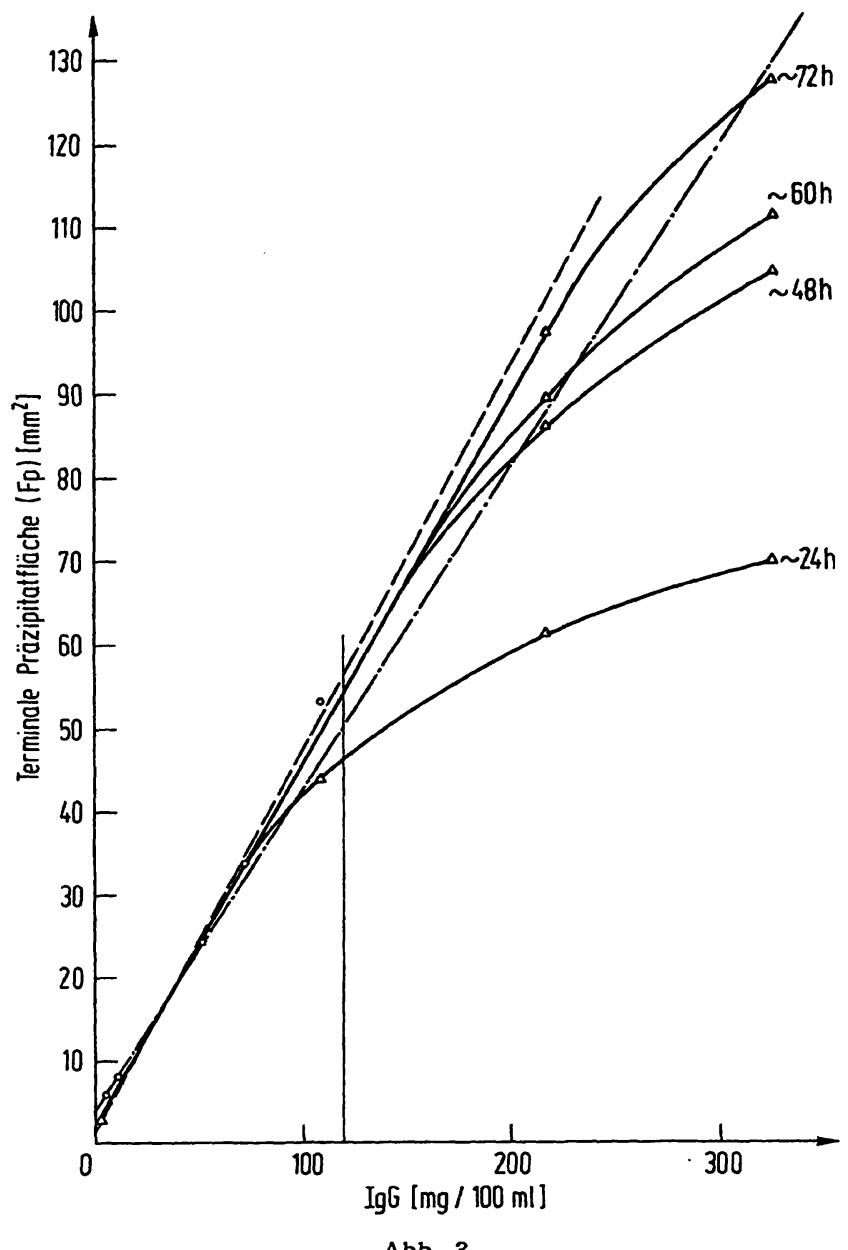

Abb. 3

Grafische Darstellung der Standardgeraden einer IgG-Tripartigenplatte (OP-Nr. 886, Fa. Behringwerke). Bestimmung der Präzipitatflächen verschiedener Standardserum-Verdünnungen in Abhängigkei von der Diffusionszeit.

Vorgegebene Regressionsgerade (-.--) y $=3,90+0,389 x$ Berechnete Regressionsgerade $(--\cdots)$ y $=2,16+0,463 x$;

Senkrechte Linie $=$ Bereich der Übereinstimmung der Regressionsgleichungen

den vorgegebenen in einem Konzentrationsbereich von $10-120 \mathrm{mg} / 100 \mathrm{ml}$ (IgG) und $10-100 \mathrm{mg} / 100 \mathrm{ml}$ (IgM) feststellen. Für IgA zeigten die 4 untersuchten Platten eine stärkere Streuung der Steigungen B.

Wir bestimmten auf 50 IgG-, 51 IgA- und 51 IgM-Tripartigen-Platten eigene Regressionsgeraden mit einem Standard-Humanserum (IgG) bzw. einem DreifachTripartigen-Standard (Standard Immunglobulin für IgA- bzw. IgM-Standard). Dabei lagen die VariationsKoeffizienten der A-Werte $z$ wischen 30 und $70 \%$, die der B-Werte (Steigung) zwischen 9,6 und 21,1\%. Unter Berücksichtigung der Platten gleicher Charge (N zwischen 21 und 26) zeigte sich keine wesentliche Änderung der Streuung beider Parameter. Aufgrund der Untersuchungen empfiehlt es sich, auch für Tripartigen-Platten Immunglobulin-Standards zur Herstellung einer Eichgeraden zu verwenden, wenn es auf größere Genauigkeit ankommt. Die Präzision der Methode für Tripartigen-Platten geht aus Tabelle 3 -hervor. Danach beträgt die Gesamt-Streuung für die untersuchten IgG-Tripartigen-Platten 3,4\% (V), für die IgA-Platten steigt sie bis $4,1 \%$, für IgM bis $6,4 \%$ an.

\section{Auswertung auf M-Partigen-Platten}

In Anlehnung an die Tripartigen-Platten sind seit kurzem M-Partigen-Platten auch für die übrigen Serumproteine im Handel. Als Beispiel untersuchten wir ein Kollektiv von 20 Haptoglobin-M-Partigen-Platten und fanden besonders niedrige Variationskoeffizienten des A- $(24,6 \%)$ und B-Wertes $(8,5 \%)$ sowie für die DreifachStandards (Tab. 3, zwischen 0,3 und 2,4\%).

\section{Auswertung auf Immuno-Plates}

Wir konnten feststellen, daß das von uns entwickelte Auswerteprogramm sich auch hervorragend zur Auswertung von Immunodiffusionsplatten (Immuno-Plate) eignet. Ein Beispiel für die grafische Darstellung der aus den Regressionsgeraden von 3-4 Platten ermittelten Gesamt-Regressionsgeraden für die Immunglobuline IgG, IgA und IgM zeigt Abbildung 4.

Wir untersuchten auf je 2-6 Immunoplates für 8 verschiedene Serumproteine die Standardseren der Fa. Hyland und Fa. Behringwerke (Standard-Humanserum stabilisiert) und bestimmten für jede Platte die aufgrund der Hyland- bzw. Behringwerke-Standardkonzentration ermittelte Standard-Regressionsgerade. Mittelwert, Streuung und Variationskoeffizient der A- und B-Werte sowie die Gleichung der Gesamt-Regressionsgerade mit Korrelations-Koeffizient und Streuung $s_{y \cdot x}$ gehen aus Tabelle 4 hervor. Außerdem sind in der letzten Spalte noch die Maximal-Abweichungen der aufgrund der Standard-Regressionsgeraden ermittelten untersuchten Standard-Konzentrationen von den angegebenen Konzentrationswerten aufgeführt. Der Tabelle ist zu entnehmen, daß die Streuungen der A-Werte (Ordinatenabschnitt) oft sehr groß sind und zwischen $3,7 \%$ und $121 \%$ (VK) schwanken, während die Variationskoeffizienten der B-Werte (Steigung) nur

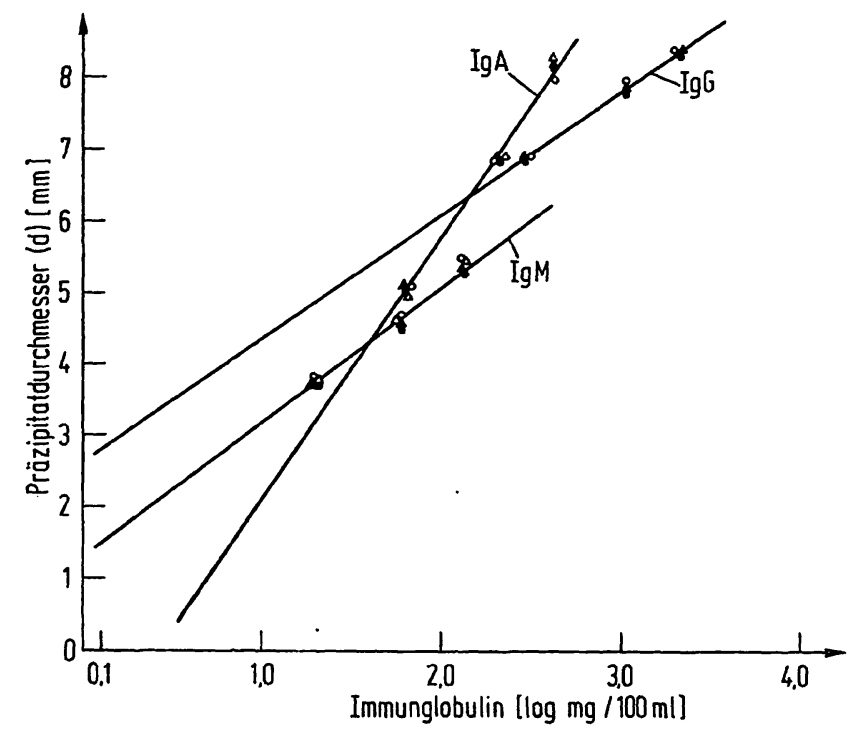

Abb. 4

Grafische Darstellung der Gesamt-Standardgeraden (lineare GesamtIII $=0, I V=A$ ) zur $\begin{gathered}\text { Bestimmung der Immunglobuline IgM, } \\ \text { IgA und IgG }\end{gathered}$ Auswertung nach der Methode von FAirey

IgA: $y=3,7 x-1,5 ; s_{y x}=0,104 r=0,997(n=4)$

IgM: $y=1,9 x+1,4 ; s_{y x}=0,109 r=0,988(n=4)$ 


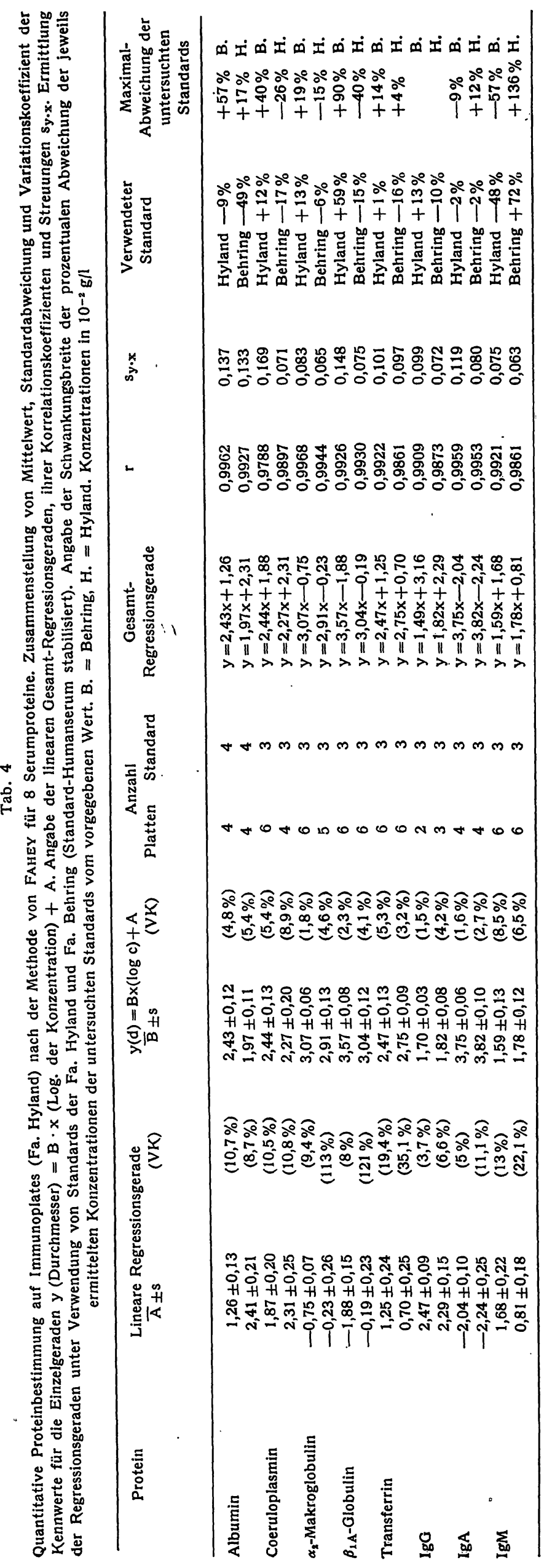

zwischen $1,5 \%$ und $8,9 \%$ streuen. Die aus bis zu 6 Einzelgeraden ermittelten Gesamtregressionsgeraden wiesen eine sehr gute Korrelation $(0,9788-0,9968)$ und relativ kleine Streuungen auf $\left(s_{y \cdot x} z\right.$ wischen 0,063 und $0,169)$.

\section{Vergleich qwischen Bebring- und Hyland-Standards}

a) Bestimmung auf Immuno-Plates

Wie aus der letzten Spalte der Tabelle 4 hervorgeht, konnten bei der Bestimmung der Standards eines Herstellers unter Verwendung der Standards des anderen Herstellers als Standardserum beträchtliche Abweichungen vom Nennwert festgestellt werden. Die Extremabweichungen von den vorgegebenen Konzentrationen der untersuchten Standards waren am stärksten ausgeprägt bei Albumin, $\beta_{1 \mathrm{~A}}$-Globulin, IgM, während sie am geringsten bei $\alpha_{2}$-Makroglobulin, Transferrin, IgG und IgA ausfielen.

\section{b) Bestimmung auf Partigen-Platten}

In einer weiteren Untersuchung wurden die HylandStandardkonzentrationen der einzelnen Proteine auf entsprechenden Partigen-Platten der Fa. Behringwerke bestimmt. Als Standard-Konzentration zur Bestimmung der Standard-Konzentrationsgeraden diente StandardHumanserum stabilisiert der gleichen Firma (Tab. 5). Wie die Tabelle zeigt, sind die Abweichungen der bestimmten Konzentrationen von dem vorgegebenen Hyland-Standard für Albumin stark wechselsinnig schwankend, für IgM am stärksten positiv (über $+50 \%$ ), für Coeruloplasmin und $\beta_{1 \mathrm{~A}}$-Globulin etwa gleichermaßen stark negativ (um $-35 \%$ ), für IgA weniger ausgeprägt negativ, während sie für Transferrin, $\alpha_{2}$-Makroglobulin und $\operatorname{IgG}$ am geringsten sind (um $\pm 15 \%$ ).

Aus dieser Zusammenstellung ist zu entnehmen, daß man die mit den beiden Plattentypen bestimmten Serumproteinkonzentrationen nicht ohne weiteres miteinander vergleichen kann. Die gefundenen Unterschiede beruhen aufgrund unserer Untersuchungen nicht auf methodischen Fehlern, sondern sind einwandfrei auf die nicht vergleichbaren Konzentrationsangaben für die Standards beider Herstellerfirmen zurückzuführen.

\section{Transformationen}

Wir haben deshalb untersucht, ob sich aufgrund der vergleichenden Untersuchungen für beide Immunodiffusionsplattentypen und die verschiedenen Standards eine lineare Beziehung $z$ wischen den jeweils bestimmten Standard-Konzentrationen (gefundener Wert $=y)$ und den angegebenen KonzentrationsWerten (Soll-Wert $=x$ ) herstellen läßt. Eine solche Beziehung müßte sinngemäß auch auf AnalysenLösungen übertragbar sein. Die so erhaltenen linearen Regressionsgeraden-Gleichungen sind zusammen mit den zugehörigen Korrelations-Koeffizienten und Streuungen $\mathrm{s}_{\mathrm{y} . \mathrm{z}}$ sowie unter Angabe der Anzahl der benutzten 
Tab. 5

Konzentrationsbestimmung der Standards von Immunoplates (Fa. Hyland) auf Partigenplatten (Fa. Behringwerke) unter Verwendung von Standards der Fa. Behringwerke (Standard-Humanserum stabilislert) aus Mehrfachbestimmungen (Anzahl $N$ ). Zusammenstellung der jeweiligen angegebenen Konzentrationen der Standards der Fa. Hyland sowie der bestimmten gemittelten Konzentration ( $\mathrm{X}$ ) mit Standard-Abweichung (s) und Variationskoeffizlenten (VK), ferner Angabe der Abweichung der bestimmten gemittelten Konzentrationswerte von den angegebenen Konzentrationen. Konzentrationen in $10^{-8} \mathrm{~g} / \mathrm{l}$. *gleiche Untersuchungen mit einem Richtigkeits-Kontrollserum der Fa. Behringwerke

\begin{tabular}{|c|c|c|c|c|c|}
\hline Protein & Bezugsgröße & Standard 1 & Standard II & Standard III & Standard IV \\
\hline \multirow[t]{2}{*}{ Albumin } & Standard/Abweichung & $(18)-25,4 \%$ & $(65)+8,6 \%$ & $(285)+41,4 \%$ & $(710)+54,2 \%$ \\
\hline & $\begin{array}{l}\bar{x} \pm s \\
\mathrm{n} / \mathrm{VK}\end{array}$ & $\begin{array}{c}13,4 \pm 0,38 \\
2 / 2,8 \%\end{array}$ & $\begin{array}{r}70,6 \pm 0,73 \\
2 / 1,0 \%\end{array}$ & $\begin{aligned} 402,9 & \pm 0,78 \\
2 & / 0,2 \%\end{aligned}$ & $\begin{array}{r}1095,2 \pm 18,87 \\
2 / 1,7 \%\end{array}$ \\
\hline \multirow[t]{2}{*}{ Coeruloplasmin } & Standard/Abweichung & $(9,5)-37,2 \%$ & $(24)-31,2 \%$ & & \\
\hline & $\begin{array}{l}\bar{x} \pm s \\
n / V K\end{array}$ & $\begin{array}{l}6,0 \pm 0,02 \\
2 / 0,3 \%\end{array}$ & $\begin{aligned} 16,5 & \pm 1,21 \\
2 & / 7,3 \%\end{aligned}$ & & \\
\hline \multirow[t]{2}{*}{$\alpha_{2}-$ Makroglobulin } & $\begin{array}{c}\text { Standard/Abweichung } \\
\overline{\mathbf{x}} \pm s\end{array}$ & $\begin{array}{l}(65)-15,6 \% \\
54,9 \pm 0,55\end{array}$ & $\begin{array}{l}(190)-2,0 \% \\
186,2 \pm 0,80\end{array}$ & $\begin{array}{l}(390)-1,7 \% \\
383,5 \pm 2,71\end{array}$ & \\
\hline & $\mathrm{n} / \mathrm{VK}$ & $3 / 1,0 \%$ & $2 / 0,4 \%$ & $3 / 0,7 \%$ & \\
\hline \multirow[t]{2}{*}{$\beta_{1 \mathrm{~A}}$-Globulin } & Standard/Abweichung & $(60)-38,9 \%$ & $(185)-37,4 \%$ & $(360)-39,1 \%$ & \\
\hline & $\begin{array}{l}\bar{x} \pm s \\
n / V K\end{array}$ & $\begin{array}{l}36,6 \pm 0,36 \\
3 / 1,0 \%\end{array}$ & $\begin{array}{r}115,9 \pm 0,12 \\
2 / 0,1 \%\end{array}$ & $\begin{array}{r}219,4 \pm 7,03 \\
3 / 3,2 \%\end{array}$ & \\
\hline \multirow[t]{2}{*}{ Transferrin } & Standard/Abweichung & $(80)+2,5 \%$ & $(240)-7,2 \%$ & $(440)+13,9 \%$ & \\
\hline & $\begin{array}{l}\bar{x} \pm s \\
n / V K\end{array}$ & $\begin{array}{r}82,0 \pm 1,08 \\
3 / 1,3 \%\end{array}$ & $\begin{array}{r}222,8 \pm 2,90 \\
2 / 1,3 \%\end{array}$ & $\begin{array}{c}500,9 \pm 3,16 \\
3 / 0,6 \%\end{array}$ & \\
\hline \multirow[t]{3}{*}{ IgG } & Standard/Abweichung & $(280)+6 \%$ & $(1000)-4,7 \%$ & $(2000)-12,3 \%$ & $(1250) *-3,7 \%$ \\
\hline & $\bar{x} \pm s$ & $296,7 \pm 1,7$ & $953,0 \pm 22,4$ & $1753,6 \pm 17,7$ & $1204,1 \pm 54,5$ \\
\hline & n / VK & $2 / 0,6 \%$ & $2 / 2,4 \%$ & $2 / 1 \%$ & $2 / 4,5 \%$ \\
\hline \multirow[t]{3}{*}{ IgA } & Standard/Abweichung & $(60)-4,8 \%$ & $(200)-19,6 \%$ & $(400)-22,2 \%$ & $(285)^{*}+3,4 \%$ \\
\hline & $\bar{x} \pm s$ & $57,1 \pm 0,41$ & $160,9 \pm 1,08$ & $311,3 \pm 2,10$ & $294,6 \pm 9,9$ \\
\hline & n / VK & $2 / 0,7 \%$ & $2 / 0,7 \%$ & $2 / 0,7 \%$ & $2 / 3,4 \%$ \\
\hline \multirow[t]{3}{*}{$\operatorname{Ig} M$} & Standard/Abweichung & $(18)+85,5 \%$ & $(58)+76,1 \%$ & $(130)+51,7 \%$ & $(115) *-6,2 \%$ \\
\hline & $\bar{x} \pm s$ & $33,4 \pm 0,48$ & $102,1 \pm 2,09$ & $197,2 \pm 1,15$ & 107,9 \\
\hline & $\mathrm{n} / \mathrm{VK}$ & $2 / 1,4 \%$ & $2 / 2,1 \%$ & $2 / 0,6 \%$ & 1 \\
\hline
\end{tabular}

Tab. 6

Transformation zwischen Standard-Konzentrationen der Fa. Behring (Partigenplatten, Standard-Humanserum stabilisiert) und der Fa. Hyland (Immunoplates) bei der einfachen radialen Immunodiffusion. Beispiel: Albumin-Hyland-Standard mit Konzentrationswert 1000 - 10-2 g/l läßt im Mittel auf einer Partigenplatte unter Verwendung des Behring-Standards einen Wert (erwarteter $=$ gefundener Wert) von $y=1,57 \cdot 1000-28,38\left(10^{-2} \mathrm{~g} / \mathrm{l}\right)$ erwarten. $\overline{\mathrm{P}}$ gemittelter Koordinatenpunkt $(\mathrm{x}, \mathrm{y})$ zur Ermittlung der linearen Regressionsgeraden

\begin{tabular}{|c|c|c|c|c|}
\hline $\begin{array}{l}\text { Verwendete Platten } \\
\text { und Standards }\end{array}$ & Albumin & $\alpha_{2}$-Makroglobulin & Transferrin & $\beta_{1 \mathrm{~A}}$-Globulin \\
\hline $\begin{array}{l}\text { Partigen-Platten } \\
\text { Standard Behring } \\
\text { y: Gefunden für Hyland } \\
\text { x: Sollwert Hyland } \\
\text { Immunoplates } \\
\text { Standard Hyland } \\
\text { y: Gefunden für Behring } \\
\text { x: Sollwert Behring }\end{array}$ & $\begin{aligned} y & =1,57 x-28,38 \\
r & =0,9996(3 \bar{P} .) \\
s_{y x} & =17,218 \\
y & =0,87 x+32,68 \\
r & =0,9999(4 \bar{P} .) \\
s_{y x} & =5,217\end{aligned}$ & $\begin{aligned} y & =1,01 x-8,70 \\
r & =0,9998(3 \bar{P} .) \\
s_{y x} & =4,051 \\
y & =1,10 x+4,43 \\
r & =0,9999(3 \bar{P} .) \\
s_{y x} & =1,104\end{aligned}$ & $\begin{aligned} y & =1,17 x-28,61 \\
r & =0,9924(3 \bar{P} .) \\
s_{y x} & =37,174 \\
y & =1,21 x-20,53 \\
r & =0,9998 \\
s_{y x} & =3,277\end{aligned}$ & $\begin{aligned} y & =0,61 x+1,36 \\
r & =0,9998(3 \bar{P} .) \\
s_{y x} & =2,526 \\
y & =1,46 x+9,63 \\
r & =0,9982 \\
s_{y x} & =3,440\end{aligned}$ \\
\hline $\begin{array}{l}\text { Verwendete Platten } \\
\text { und Standards }\end{array}$ & $\operatorname{IgG}$ & IgA & $\lg M$ & Coeruloplasmin \\
\hline $\begin{array}{l}\text { Partigen-Platten } \\
\text { Standard Hyland } \\
\text { y: Gefunden für Behring } \\
\text { x: Sollwert Hyland } \\
\text { Immunoplates }\end{array}$ & $\begin{aligned} y & =0,90 x+48,52 \\
r & =0,9965(6 \bar{P} .) \\
s_{y x} & =66,285\end{aligned}$ & $\begin{aligned} y & =0,72 x+6,86 \\
r & =0,9930(6 \bar{P} .) \\
s_{y x} & =14,903 \\
y & =0,96 x-4,0\end{aligned}$ & $\begin{aligned} y & =1,45 x+11,63 \\
r & =0,9975(3 \bar{P} .) \\
s_{y x} & =8,225 \\
y & =0,57 x-7,10\end{aligned}$ & $y=0,96 x+3,83$ \\
\hline $\begin{array}{l}\text { Standard Hyland } \\
\text { y: Gefunden für Behring } \\
\text { x: Sollwert Behring }\end{array}$ & & $\begin{aligned} r & =0,9994(3 \text { P. }) \\
s_{y x} & =4,624\end{aligned}$ & $\begin{aligned} r & =0,9963(3 \bar{P} .) \\
s_{y x} & =2,778\end{aligned}$ & $\begin{aligned} r & =0,9948\left(3 \bar{P}_{.}\right) \\
s_{\mathrm{yx}} & =1,060\end{aligned}$ \\
\hline
\end{tabular}

Wertepaare $(\mathrm{x} ; \mathrm{y})$ in Tabelle 6 zusammengestellt. Alle Geraden besitzen einen Korrelations-Koeffizienten von über 0,99. Die Geradenstreuungen sind unterschiedlich groß und schwanken zwischen 1,060 und in einem extremen Fall 66,285 (IgG).
Aufgrund der gefundenen Werte lassen sich anhand der Tabelle für die einzelnen Proteine und Plattentypen sowie bei Verwendung der einzelnen Standards die zu erwartenden Konzentrationen mit einer oft erheblichen Schwankungsbreite abschätzen. 


\section{Literatur}

1. Mancini, G., Carbonara, A. O. \& Heremans, J. F. (1965), Elsevier-Amsterdam. - 4. Fateh-Moghadam, Lamerz, R., Immunochenistry 2, 235-254. - 2. FAhex, J. F. \& McKelvex, Eisenburg, J. \& Kneded, M. (1969), Klin. Wochenschr. 47, E. M. (1965), J. Immunol. 94, 84-90. - 3. Augener; W. (1965), Immunanalyse von Glykoproteinen. In: H. PeEters (Ed.): Proc. XIIth Coll. Protides of the Biolog. Fluids, Brïgge 1964, 363-371, 129-140. - 5. SACHS, L. (1968), Statistische Auswertemethoden. Springer Verlag Berlin-Heidelberg-New York.

Dr. R. Lamerz .

Priv. Doz. Dr. A. Fateh-Moghadam

1. Medizinische Klinik der Universität München 8000 München 2

Ziemssenstr. 1a

Priv. Doz. Dr. M. Knedel

Klinisch-Chemisches Institut am Städt. Krankenhaus München-Harlaching

8000 München 90

Sanatoriumsplatz 2 